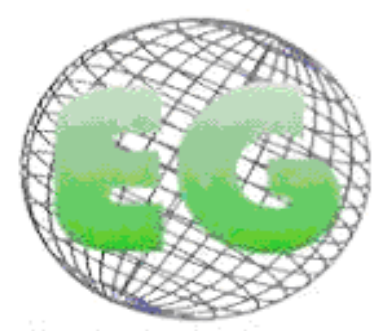

ISSN 1695-6141 N 25

\title{
Percepción del familiar acompañante con respecto al cuidado de enfermería en hospitalización
}

Perception of family companion with respect to nursing care in hospitalization

\author{
*Ramos Frausto, VM., "Rico Venegas, RM., "Martínez, PC. \\ *Departamento de Enfermería y Obstetricia. Universidad de Guanajuato. Campus de León. Mexico.
}

Palabras clave: percepción del familiar; cuidado de enfermería; hospitalización.

Keywords: perception of family; nursing care; hospitalization

\section{RESUMEN}

Antecedentes: La atención a la salud es considerada a nivel mundial como uno de los servicios con mayor demanda social. La percepción del familiar acompañante se guía por ciertas expectativas, que se ven modificadas a consecuencia de algunas actividades realizadas por el personal de Enfermería, reflejando el grado de satisfacción de los servicios otorgados.

Objetivo: Evaluar la percepción del familiar acompañante con respecto al cuidado que otorga el personal de Enfermería durante su estancia hospitalaria.

Material y Métodos: Estudio descriptivo, prospectivo de corte transversal. En el periodo mayo-junio de 2010. El tamaño de la muestra se determinó considerando un nivel de confianza de 0.5 , una potencia de prueba del 95\% que dio un tamaño de muestra de 153 participantes.

Resultados: Los resultados con respecto a la atención de Enfermería se analizaron para determinar si existía alguna asociación entre las diferentes variables, determinando que no existía asociación significativa, en nuestro hospital, se presentó $23.5 \%$ de insatisfacción tomando en cuenta las áreas, iniciando con la oportunidad del $4.12 \%$, la amabilidad, un $4.24 \%$ en el proceso de la atención $4.31 \%$, el resultado de la atención, señalando un $4.47 \%$ con respecto a la relación enfermera-paciente, de igual forma se encontró un $4.69 \%$ con respecto a la información otorgada sobre su cuidado $4.25 \%$. Encontrando en la comodidad otorgada al paciente el $3.68 \%$ se observó que el índice afectado es la atención en la comodidad

\section{ABSTRACT}

Background: Health care is regarded worldwide as one of the social services most in demand. The perception of family caregivers is guided by certain expectations, which are modified as a result of certain activities undertaken by the nursing staff, reflecting the degree of satisfaction with services rendered.

Aim: To assess the perception of family caregivers regarding the care given by nursing staff during their hospital stay. 
Material \& Methods: A prospective descriptive cross-sectional study performed in the period May to June 2010. The sample size was determined considering a confidence level of 0.5 and a test power of $95 \%$, which gave a final sample size of 153.

Results: The results with respect to nursing care were examined to determine whether there was any association between different variables, determining that there was no significant association in our hospital. $23.5 \%$ of dissatisfaction was found, taking into account the areas, starting with opportunity $(4.12 \%)$; kindness $(4.24 \%)$; the care process (4.31\%); the result of the attention $4.47 \%$; for the nurse-patient relationship, just as we found a $4.69 \%$ over information about the care given $4.25 \%$. Comfort given to the patient was $3.68 \%$ it was observed that the affected index is comfort care.

\section{ANTECEDENTES}

La atención a la salud es considerada a nivel mundial como uno de los servicios con mayor demanda social. Y los que ofrece Enfermería corresponden a uno de los de mayor cobertura en todos los niveles de atención, de esta forma Enfermería tiene como finalidad ofrecer servicios asistenciales efectivos con sentido humanitario dirigidos al cuidado de la salud de la persona, familia y comunidad, con acciones de promoción, prevención, curación y rehabilitación otorgadas por personal profesional competente, que sustenta su práctica en una sólida formación académica centrada en el dominio de la disciplina y en los valores éticos de la profesión ${ }^{(1)}$.

La percepción del familiar acompañante, con relación a los servicios que presta el personal de Enfermería, es considerada como una actividad guiada por ciertas expectativas que pueden ser modificadas por la información que se obtiene como consecuencia de esta actividad la cual refleja en gran medida el grado de satisfacción que el familiar acompañante además del paciente pueden tener de la atención recibida; la percepción, por otro lado, depende de la calidad de los servicios otorgados. ${ }^{(1)}$

La calidad de la atención aplicada a los servicios de salud se inició durante el procedimiento del censo de Estados Unidos de América, y que confiere a todos los trabajadores de las Instituciones una mayor responsabilidad y compromiso para realizar a la perfección su labor y prestar un servicio que satisfaga las necesidades de todos los usuarios y equipo multidisciplinario. ${ }^{(2)}$

A nivel nacional, el Instituto Mexicano del Seguro Social (IMSS) es una Institución con amplia cobertura de derechohabientes, por lo que la calidad del servicio que brinda es importante al considerar el estado de salud en México. En estudios previos, como el realizado por Puebla-Viera (2009), se ha referido que el IMSS, considerada como una empresa que presta atención de salud, no está exenta de que su personal, al igual que en otras empresas, guíe sus labores diarias en base a metas propuestas por la misma, enfocando la atención en las necesidades de esta última, dejando en segundo término las necesidades del paciente y del familiar acompañante, ofreciendo un servicio de cantidad y no de calidad ${ }^{(2)}$; situación que se ha señalado es influenciada por el modelo económico del país, en donde la sociedad cada vez más globalizada está inmersa con problemas sociales como la escasez de valores e indiferencia por el prójimo. Por otro lado, se considera de suma importancia el papel que desempeña la familia dentro de la sociedad, señalando que no se pueden separar las necesidades del paciente con su familia por ser una unidad funcional, principalmente durante los procesos de enfermedad ${ }^{(2,3)}$

Por lo expuesto anteriormente, es importante para el personal de Enfermería, como parte del equipo multidisciplinario de la Unidad Médica de Alta Especialidad No. 1 Bajío, conocer la 
percepción de los familiares, ya que evalúan elementos importantes en los indicadores de calidad por la atención brindada en los diferentes servicios de hospitalización, como a continuación se plantea en el presente estudio.

\section{MARCO DE REFERENCIA}

Actualmente, la satisfacción de los usuarios es utilizada como un indicador para evaluar las intervenciones de los servicios de salud, pues nos proporciona información sobre la calidad percibida en los aspectos de estructura, procesos y resultados sobre la atención prestada en cada uno de sus servicios. Estos indicadores no son ajenos al Instituto Mexicano del Seguro Social ya que con base en ellos se puede identificar a qué nivel de calidad se está proporcionando el servicio. Enfermería se encuentra inmersa en estos cambios, ya que este personal es uno de los principales otorgadores del cuidado en los hospitales. ${ }^{(2)}$

El cuidado que ofrece una Enfermera a una persona sana o enferma es formalizado y con fundamentación científica, sistematizado a través de un plan de intervención con el objetivo de conducir a un bienestar físico y mental. La razón de ser de Enfermería es proporcionar los cuidados para el bienestar del individuo, su recuperación y mantenimiento de la salud o de otra forma ayudar a morir con dignidad. Por lo tanto, un momento de crisis en el núcleo familiar es tener a un miembro de la familia críticamente enfermo o simplemente con deterioro de su salud; de igual manera, cuando queremos entender al sujeto tenemos que partir de su situación social, como un ser humano miembro de una familia y una comunidad $^{(4,5)}$. En el momento que surge esta necesidad de asistencia social, el familiar acompañante se vuelve parte del equipo multidisciplinario en el cuidado del familiar enfermo esto dentro de un espacio adecuado en el que se pueda brindar atención directa y de calidad (hospitalización) con el fin de satisfacer sus necesidades.

Donabedian (1991) define la calidad de la atención médica como la atención que depende tanto de la percepción como del conocimiento, y se basa tanto en las percepciones del paciente y familiar como en la síntesis exacta de las observaciones de la medicina moderna $^{(6)}$.

El hospital resulta ser un lugar hostil tanto para el enfermo como para el familiar acompañante, en donde se encuentran solos, asustados, con miedo, donde deben adaptarse a un medio extraño y a un lenguaje especializado, e incluso se encuentran en ciudades y hospitales desconocidos, dependiendo de personas que no conocen y enfrentándose a problemas que no comprenden ${ }^{(1)}$, necesitando de manera imperante comunicarse con los demás, planteado como un desafío que provoca estrés de carga que afecta su salud física y mental, percibiendo el cuidado proporcionado por el personal de salud de diferentes formas desde una forma satisfactoria hasta agresiva ${ }^{(7)}$

Dentro de las funciones de Enfermería está la de establecer asistencia utilizando herramientas psicológicas como la relación de ayuda, para favorecer la disminución de la ansiedad en el primer contacto de hospitalización de los pacientes así como su familiar acompañante. Los conceptos teóricos que fundamentan la relación de ayuda fueron desarrollados por teoristas como Peplau H, Orlando I, y Travelbee J (1990). Peplau en su modelo de Enfermería psicodinámica aborda un tipo de relación Enfermera-paciente que se conoce como relaciones interpersonales; en la cual las dos personas llegan a conocerse lo suficientemente, como para afrontar los problemas que surjan de manera cooperativa.

Orlando por lo tanto considera que la función de Enfermería es averiguar y satisfacer las necesidades de ayuda inmediata al paciente. Esta función se logra cuando la Enfermera 
satisface esas necesidades a través de los cuidados de Enfermería. Travelbee J, en su modelo de persona a persona, establece que el propósito de Enfermería es ayudar al individuo, familia y comunidad a prevenir o enfrentar la experiencia que supone la enfermedad y si es necesario encontrarle un sentido a dichas experiencias. ${ }^{(8)}$ Cuando la Enfermera adopta una actitud cálida, de respeto, comprensión y empatía (enfoque de relación de ayuda) brinda una atención integral y logra disminuir la ansiedad situacional de los familiares y de los pacientes. ${ }^{(1)}$

En este sentido, la Enfermera es un punto fundamental en la recuperación de la salud del mismo, quien al sentirse más confiado, puede colaborar más efectivamente en su recuperación. ${ }^{(2)}$ Para ello la comunicación es fundamental para lograr el éxito de la relación interpersonal, así como la confianza que se expresa a través de una sonrisa y un lenguaje sencillo el cual ayuda a clarificar el mensaje y profundizar en la esfera psicológica de la carga de afectividad y significados que tiene la palabra, así la interacción se refiere a cualquier contacto entre dos individuos durante el cual se ejerce una influencia recíproca y entre sí una comunicación verbal o no verbal. (9) Por tanto la Enfermera debe agudizar sus sentidos y desarrollar su capacidad de escuchar, observar la comunicación no verbal y el lenguaje emocional del paciente. La Enfermera requiere de competitividad profesional, múltiples habilidades y relación armónica, con el familiar y el paciente, por lo tanto, es importante que tome en cuenta que a diario trasmite un impacto mediante su comunicación.

En la comunicación interpersonal es conectarse con otras personas en un nivel emocional y no solo en un nivel intelectual. ${ }^{(9)}$ En el caso específico de la relación familiar, Enfermera y paciente refieren López y Varga (2002) que las nueve habilidades que pueden coadyuvar a enriquecer la importante labor que desempeña enfermería son: El comportamiento visual, Postura y movimiento, Gestos y expresión, El vestir y el aspecto, Voz y variedad vocal, El lenguaje, La atención, El humor, Naturalidad. Estas nueve habilidades son percibidas por los familiares, en relación a los cuidados que brinda el profesional de Enfermería a sus pacientes y es considerado de suma importancia dentro del trabajo que realiza este personal, ya que además de contribuir con en el tratamiento y recuperación, por ende, el ambiente hospitalario le sea lo menos hostil o desagradable posible.

Novaes y colaboradores (1999) señalan que la percepción es el primer proceso cognoscitivo, a través del cual los familiares captan la información del entorno, llevándola a sus sistemas sensoriales formándose una representación de la realidad del cuidado ${ }^{(10)}$. El proceso de la percepción, se propone que es de carácter inferencial y constructivo, el cual genera una representación interna de lo que sucede en el exterior al modo de hipótesis. Para ello se usa la información que llega a los receptores y se va analizando paulatinamente, así como información que viene de la memoria tanto empírica, como genética y que ayuda a la interpretación y a la formación de la representación del cuidado ${ }^{(2)}$

La percepción varía de acuerdo a que experiencia y a que aprendizaje previo tenga el familiar. Los aspectos que influyen al medir la satisfacción laboral sobre la persona que percibe son:

1. Las necesidades y deseos, es decir, la motivación de la persona la cual le hace percibir aquello que le proporcionaría satisfacción.

2. Las expectativas; se tiende a percibir lo que se espera, aquello que resulta más familiar.

3. El estilo de cada persona para enfrentarse al ambiente que lo rodea. Algunas personas perciben más un conjunto de detalles, pudiendo recordar por separado algunas características, otras en cambio reparan en tales detalles. 
4. La cultura en la que creció, la cual entrena en cierto modo de percibir la realidad ${ }^{(6,10,11)}$

Toda la información y los estímulos que se captan por los sentidos, más aquellos aspectos que influyen en la forma de percibir, generan la elaboración de un concepto. La percepción del familiar con respecto a la intervención de Enfermería durante su hospitalización puede variar de acuerdo a los puntos antes mencionados.

Los estudios enfocados al familiar acompañante realizados dentro de las instituciones son escasos, esta situación determina el no reconocimiento por parte de los sistemas de salud, ya que para ellos pasan desapercibidos, por lo que en líneas generales, el servicio de salud es incapaz de satisfacer.

Entendemos por familiar acompañante aquellos que son familia, amigos, vecinos, compañeros de trabajo u otras personas. Los elementos básicos que lo definen son: su carácter no institucional, la no remuneración. Ya que se desarrollan en el terreno de lo privado, asentados sobre relaciones personales generalmente de parentesco. También asumen la responsabilidad de atender las necesidades de la persona hospitalizada.

Esto se traduce en una presencia física casi permanente en el entorno hospitalario, entonces debemos considerar que los familiares son una parte fundamental en la recuperación y el cuidado del enfermo. El objetivo primordial dentro de los servicios de salud es la calidad de la atención, otorgando una mayor responsabilidad y compromiso a todo el equipo, multidisciplinario para satisfacer las necesidades de todos y mantener elevados nuestros indicadores de calidad.

\section{JUSTIFICACIÓN.}

La Organización Mundial de la Salud (OMS, 2009) en su declaración de los Derechos Humanos en su Artículo 3ro menciona que "Todo individuo tiene derecho a la vida, a la libertad y a la seguridad de su persona". En nuestro país, la Constitución Política Mexicana en su Artículo 4to. menciona en el párrafo 3ero que "Toda persona tiene derecho a la protección de la salud", tomando al humano como un ser holístico, y miembro de una familia, la cual es el principal apoyo, en una necesidad de salud".

Al tener la necesidad de integrarse a un medio hospitalario, los familiares como se ha referido previamente, pasan desapercibidos para el sistema ya que realizan una actividad, que en líneas generales, los servicios de salud es incapaz de satisfacer porque corresponde a las emociones y afectos. Si bien los estudios del familiar acompañante han sido ampliamente abordados dentro del ámbito de la atención primaria y en el entorno domiciliario en relación con determinadas patologías $(9,2)$. Existen pocos estudios $(1,6,10)$ que incidan sobre su realidad dentro de los servicios de salud en hospitalización, de ahí, la importancia de esta investigación.

La UMAE No. 1 Bajío es una Institución de concentración en donde actualmente se cuenta con 231 camas censables y si tomamos en cuenta que por cada paciente existe un familiar acompañante, podemos darnos cuenta de que en el transcurso del periodo que se realizara el presente estudio tendremos la participación de un número muy significativo de familiares participantes, ya que para el Instituto Mexicano del Seguro Social la atención y satisfacción del usuario es lo más importante porque se encuentra ligada con el trato del profesional de Enfermería. Hasta hoy día no se han realizado estudios en donde se evalúen estos estándares de calidad con respecto a la percepción del familiar acompañante por lo que es importante el desarrollo del presente estudio. 


\section{PLANTEAMIENTO DEL PROBLEMA}

¿Cuál es la percepción del familiar acompañante con respecto al cuidado que otorga el personal de Enfermería en los servicios de hospitalización de la UMAE no.1 Bajío?

\section{OBJETIVO GENERAL.}

Evaluar la percepción del familiar acompañante con respecto al cuidado que otorga el personal de enfermería durante su estancia hospitalaria.

\section{Objetivos específicos.}

1. Conocer el grado de satisfacción del familiar acompañante de los cuidados de Enfermería.

2. Identificar la proporción de pacientes hospitalizados que cuentan con familiares acompañantes.

\section{DISEÑO}

Se realizó un estudio descriptivo, prospectivo, de corte transversal, en el que se midió la percepción de los familiares acompañantes, respecto a la atención recibida por el personal de Enfermería de la Unidad Médica (UMAE) no. 1 Bajío.

\section{MATERIALES Y METODOS}

\section{Universo}

La población considerada estuvo compuesta por los participantes que permanecieron al cuidado de su familiar hospitalizado en cada una de las 231 camas censables en el hospital UMAE №1 Bajío en el periodo de abril a mayo del año 2010. Para calcular el tamaño de la muestra se utilizó el paquete estadístico MedCalc 9,2 para estimación de proporciones referido por Jiménez y Villegas $\mathrm{M}$, et al (2003) ${ }^{(13)}$ con grado de insatisfacción de un $28 \%$, el cual se espera que en nuestro hospital este se presente en un $15 \%$ similar al que reporta Ortiz Espinoza R, et al (2003) ${ }^{(14)}$, considerando un nivel de confianza de $95 \%$, error tipo I aceptado alfa a de 0.05 , una potencia de $80 \%$ y asumiendo un $20 \%$ por los posibles participantes que no contesten completo el cuestionario, se obtuvo un tamaño de la muestra de 153 participantes, para evaluar la calidad de la atención de Enfermería se utilizó el instrumento descrito por Ponce Gómez y colaboradores (2006) ${ }^{(12)}$,

Este evalúa siete áreas (oportunidad, amabilidad, proceso de la atención, resultados, relación enfermera-paciente, información y comodidad) el cual consta de 7 ítems evaluado con cinco reactivos de respuesta tipo likert catalogadas de mayor a menor grado de satisfacción, este obtuvo una Alpha de Cronbach de 0.8492 lo cual mostró la consistencia interna de sus ítems en un rango de 1 a 5 . El muestreo se realizó por conveniencia por numeración de camas pares e impares y por turno matutino y vespertino, determinando 77 para el turno matutino y 76 para el turno vespertino, estará compuesto por hombres y mujeres entre 18 y 60 años de edad. Sólo participaron los familiares acompañantes que permanezcan en hospitalización durante tres días acompañando a su paciente en cualquiera de los dos turnos. Posterior a la selección de los familiares, se recopiló la información a través del instrumento de medición. 


\section{Criterios de selección.}

Criterios de inclusión.

1. Todos los familiares acompañantes de pacientes hospitalizados que se encuentren presentes en el hospital UMAE \#1 Bajío en el periodo de abril a mayo del 2010.

2. Familiares acompañantes que hayan permanecido con su paciente, durante una estancia de tres días y por lo menos en alguno de los dos turnos.

\section{Criterios de exclusión.}

1. Acompañante que perciba remuneración económica.

2. Familiar que se niegue a contestar la encuesta.

\section{DEFINICIÓN DE VARIABLES}

VARIABLE INDEPENDIENTE: Cuidado de Enfermería

VARIABLE DEPENDIENTE: Percepción del familiar acompañante

\section{Descripción de procedimiento.}

Se contó con la aprobación del Comité de Ética e Investigación del Hospital UMAE \# 1 Bajío del IMSS en León Guanajuato, después de esto se formalizo el permiso correspondiente para realizarse el estudio. Enseguida se tomaron las listas de las camas seleccionadas por cada uno de los pisos $1^{\circ}, 2^{\circ}, 3^{\circ}$ y $4^{\circ}$ piso respectivamente. Enseguida se procedió a determinar cuáles camas se visitaron en el turno matutino y vespertino. Posteriormente se les invitó a participar y si aceptaron se les asignó el lugar para el llenado de los cuestionarios; si alguno de los participantes que fueron seleccionados no desean participar se invitará en su lugar al familiar acompañante de la siguiente cama que apareciera después de él en la lista.

El lugar de aplicación de los cuestionarios será un lugar fuera del sitio donde se encuentre el familiar hospitalizado, se hará en la sala de espera de cada uno de los pisos, el horario se determinará de manera que no interfiera con los cuidados de su paciente. El levantamiento de los datos se llevó a cabo por el investigador principal y colaboradores. Después de aplicar el instrumento, se verifico que no se hayan dejado en blanco alguna respuesta. Al final del llenado del cuestionario se les dio las gracias por su participación. Se planteó la utilización de un instrumento validado previamente el cual se describe a continuación. Para medir la calidad de la atención de Enfermería en la Unidad Médica de Alta Especialidad del IMSS UMAE \#1 Bajío, se utilizo el instrumento descrito por Ponce Gómez y colaboradores $(2006)^{(12)}$, este consta de 7 ítems evaluado con cinco reactivos de respuesta tipo Likert catalogadas de mayor a menor grado de satisfacción, el cual obtuvo una Alpha de Cronbach de 0.8492 mostrando una consistencia interna de sus ítems en un rango de 1 a 5 . Este se calificó realizando la sumatoria del valor otorgado a cada uno de los ítems, enseguida esta sumatoria se dividió entre los siete ítems del instrumento, Otorgándoles una calificación para determinar la percepción que tiene el familiar acompañante con respecto a la atención de enfermería. Donde la calificación de 5 significara excelente atención mientras que 4 suficiente atención y 3 o menos atención negativa, se catalogó 3 como insuficiente, 2 no realizada y 1 inadecuada Un ejemplo de estas preguntas sería: ¿La atención que le otorgó la Enfermera fue? 5.-Excelente 4.-Aceptable 3.-Insuficiente 2.-No le atendieron 1.-Le causó molestias. 


\section{Análisis estadístico}

A continuación se presentan los resultados del estudio; cuyo propósito fue determinar la percepción del familiar acompañante con respecto a la atención de Enfermería. Primero se muestran datos descriptivos que caracterizan a los participantes del estudio, posteriormente se muestran los resultados para responder a los objetivos.

En la gráfica \#1 se muestra los datos por grupo de edad, la cual estuvo conformada por 153 participantes, donde la edad promedio de la población oscilaba entre los 31 y 50 años, esta muestra de los participantes representó el $50.3 \%$ del total de la población ( $f=77$ ).

\section{Grafica \# 1}

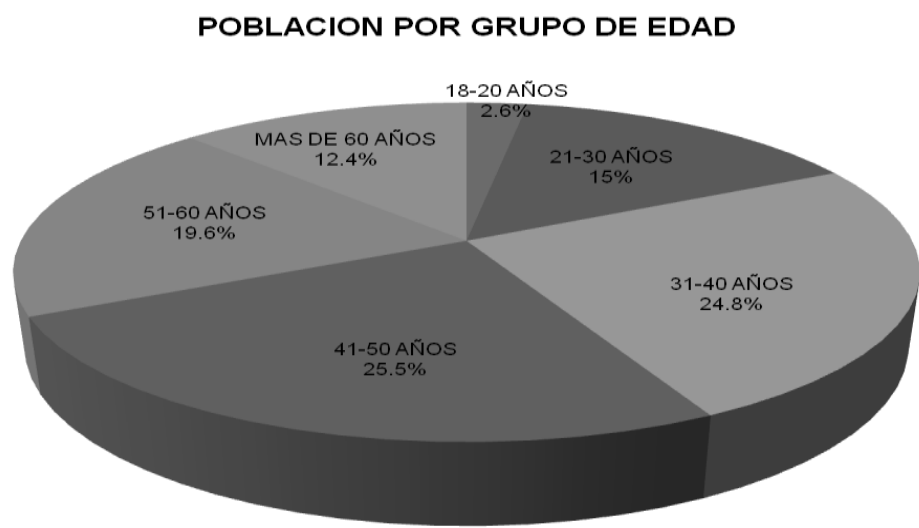

Fuente: IPMSP $\quad n=153$

En la gráfica \#2 se muestran los datos de los participantes con respecto a su estado civil, se puede observar que el $71.9 \%$ de los participantes refirieron estar casados $(f=110)$.

\section{Gráfica \#2}

\section{ESTADO CIVIL}

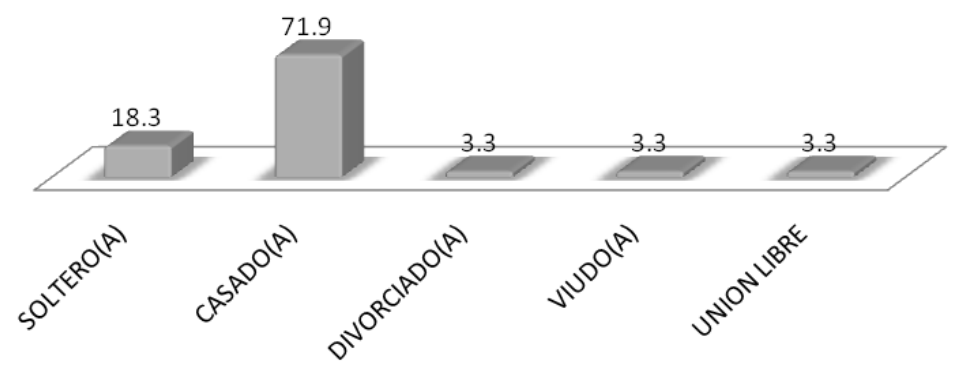

Fuente: IPMSP

$n=153$ 
Con respecto a la escolaridad que señalaron tener los participantes del presente estudio, ILos resultados se muestran en la gráfica \# 3 reportando que el nivel de primaria se presentó en un $41.2 \%(f=63)$ seguido por un $34.6 \%(f=53)$ para el nivel escolar de secundaria

\section{Gráfica \#3}

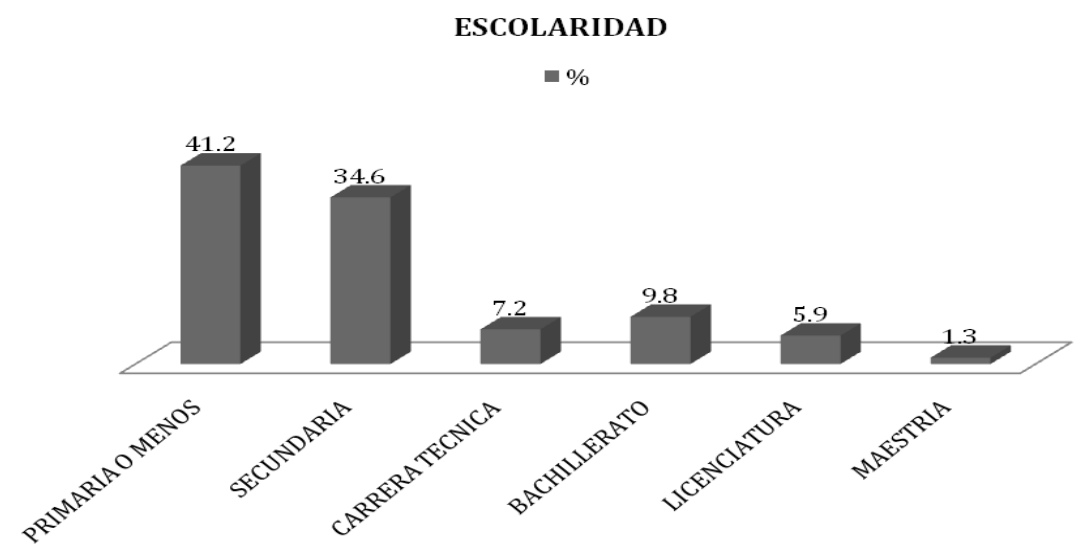

Fuente: IPMSP

$n=153$

En el presente estudio el objetivo principal fue analizar la percepción del familiar acompañante con respecto a la atención que otorga el personal de enfermería, se analizaron los datos para determinar si existía alguna asociación entre las diferentes variables.

Se analizó entre la variable por turno, matutino y vespertino, con respecto a la atención que brinda el personal de enfermería tomando un rango de calificación con una puntuación de 3 , considerando la puntuación de 4 y 5 como buena y la puntuación de 3 como mala. Encontrándose que no hubo relación significativa entre la percepción del familiar acompañante para determinar la satisfacción en el cuidado de enfermería con respecto a los turnos mencionados.

Con relación a la percepción del familiar sobre la calidad de la atención de enfermería se reportó que no hubo asociación significativa en los diferentes pisos. Sin embargo es importante destacar la diferencia en el porcentaje de estos, ya que en la aplicación del instrumento se identificaron discrepancias en la atención de Enfermería, obteniéndose los siguientes resultados. 
Tabla I

Percepción del familiar acompañante sobre la atención recibida en hospitalización

\begin{tabular}{|c|c|c|c|c|c|c|c|}
\hline piso & Oportunidad & Amabilidad & $\begin{array}{l}\text { Proceso } \\
\text { de atención }\end{array}$ & $\begin{array}{l}\text { Resultado de } \\
\text { la atención }\end{array}$ & Relación & Información & Comodidad \\
\hline 1 & 4.69 & 4.54 & 4.54 & 4.66 & 4.78 & 4.51 & 4.03 \\
\hline 2 & 4.52 & 4.38 & 4.43 & 4.68 & 4.70 & 4.45 & 3.77 \\
\hline 3 & 4.13 & 4.27 & 4.29 & 4.43 & 4.62 & 4.21 & 3.78 \\
\hline 4 & 3.17 & 3.82 & 4.00 & 4.10 & 4.66 & 3.84 & 3.20 \\
\hline Total & 4.12 & 4.24 & 4.31 & 4.47 & 4.69 & 4.25 & 3.68 \\
\hline & \multicolumn{2}{|c|}{ Fuente: IPMSP } & \multicolumn{2}{|c|}{$n=153$} & \multicolumn{3}{|c|}{$P=0.71$} \\
\hline
\end{tabular}

En la tabla 1 se pueden observar los datos que muestran cómo se presentó la atención de enfermería en los diferentes pisos del hospital UMAE \#1 Bajío, se observa que hubo una variación significativa con respecto a la atención de enfermería en el cuarto piso, de acuerdo a los ítems evaluados, en donde se menciona de qué forma percibe la atención de enfermería el familiar acompañante, señalando que se recibió tarde y a disgusto, mientras que otros participantes mencionaron haberla recibido después de 2 minutos; otro grupo mencionó haber recibido la atención de manera indiferente y seria, así también percibiéndola con falta de amabilidad, señalando también que la información que recibieron sobre los cuidados que debería tener su paciente fueron superficiales, otro factor que percibió el familiar acompañante fue la comodidad del paciente, señalando que ocasionalmente se les realizó el aseo en su cama y en su persona.

Tabla II

Percepción del familiar acompañante del servicio de enfermería

\begin{tabular}{c|c} 
Piso ( hospitalización) & Percepción Mala \\
\hline 1 & $9.0 \%$ \\
\hline 2 & $15.9 \%$ \\
\hline 3 & $21.6 \%$ \\
\hline 4 & $46.15 \%$ \\
\hline
\end{tabular}

Fuente: IPMSP

$n=153 \quad p=0.71$

Estos factores al concentrarse en el análisis estadístico no fueron significativos pero representan un porcentaje de transcendencia en la percepción del cuidado en enfermería proporcionada por piso, reportando una buena atención en un $53.84 \%$ y una mala atención percibida de un $46.15 \%$.

En lo que se refiere con la variable edad no se encontró un valor significativo para percibir la calidad de atención de enfermería por el familiar acompañante. Por otra parte se reporta que 
en estado civil. Los familiares acompañantes que se encontraban casados percibieron que la atención enfermería fue satisfactoria mostrando una asociación significativa con un valor de $X^{2} 6.01$ y una $P=.01$

La escolaridad como variable independiente no fue significativa para la percepción en la atención d enfermería. Por otro lado los años de asegurado no tuvieron peso alguno sobre la percepción del familiar acompañante, reportando una media de 17 años.

Se encontró que la percepción de los familiares no varía con relación a si tuvieran un 1 año o hasta 39 años de estar asegurado, de la misma manera se señaló con los días de estancia que no influyó en su percepción de la atención de enfermería así tuvieran tres o hasta más de días de estancia hospitalaria. Tomando en cuenta las áreas que se evaluaron en el cuidado Enfermero por medio de los ítems, se reporta un índice de satisfacción en el área de oportunidad del $4.12 \%$ para la amabilidad en la atención de enfermería un $4.24 \%$ en el proceso de la atención $4.31 \%$ para el resultado de la atención, señalando un $4.47 \%$ con respecto a la relación enfermera-paciente de igual forma se encontró un 4.69\%, con respecto a la información otorgada sobre su cuidado $4.25 \%$. Encontrando en la comodidad otorgada al paciente el 3.68\%. Se pudo observar que el índice más afectado es la atención en la comodidad, factor importante para el bienestar del paciente, ya que se ve afectado por carencias de recursos y se puede mencionar que en lo que refiere en este tema, que esta es una actividad compartida con el equipo multidisciplinario del hospital y no le compete solo al personal de Enfermería. Tabla \#3.

Tabla \# 3

\begin{tabular}{|c|c|c|c|c|c|c|c|c|c|c|}
\hline Variable & $\begin{array}{c}\text { Escala de } \\
\text { variable }\end{array}$ & Frec. & $\begin{array}{c}\text { Indice de } \\
\text { satisfacción }\end{array}$ & Oportunidad & Amabilidad & $\begin{array}{c}\text { Proceso de } \\
\text { atención }\end{array}$ & $\begin{array}{l}\text { Resultados } \\
\text { De atención }\end{array}$ & $\begin{array}{c}\text { Relación } \\
\text { Enf-pte }\end{array}$ & Información & Comodidad \\
\hline \multirow[t]{2}{*}{ Turno } & Matutino & 77 & 4.22 & 4.10 & 4.25 & 4.25 & 4.40 & 4.75 & 4.28 & 3.54 \\
\hline & Vespertino & 76 & 4.27 & 4.10 & 4.23 & 4.36 & 4.53 & 4.64 & 4.22 & 3.82 \\
\hline \multirow{6}{*}{ Edad } & $18-20$ & 4 & 4.21 & 3.75 & 4.00 & 4.50 & 4.00 & 4.75 & 4.00 & 4.50 \\
\hline & $21-30$ & 23 & 4.17 & 3.91 & 4.26 & 4.26 & 4.26 & 4.52 & 4.08 & 3.95 \\
\hline & $31-40$ & 38 & 4.17 & 3.92 & 4.15 & 4.23 & 4.39 & 4.68 & 4.23 & 3.65 \\
\hline & $41-50$ & 39 & 4.21 & 4.10 & 4.15 & 4.35 & 4.56 & 4.79 & 4.20 & 3.33 \\
\hline & $51-60$ & 30 & 4.43 & 4.46 & 4.50 & 4.40 & 4.60 & 4.66 & 4.50 & 3.90 \\
\hline & 600 mas & 19 & 4.30 & 4.36 & 4.26 & 4.26 & 4.57 & 4.78 & 4.26 & 3.63 \\
\hline \multirow{6}{*}{ Escolaridad } & Primaria & 63 & 4.32 & 4.33 & 4.38 & 4.41 & 4.52 & 4.71 & 4.28 & 3.63 \\
\hline & Secundaria & 53 & 4.24 & 4.05 & 4.13 & 4.24 & 4.47 & 4.79 & 4.37 & 3.67 \\
\hline & Est técnicos & 11 & 4.35 & 4.27 & 4.36 & 4.36 & 4.72 & 4.72 & 4.54 & 3.54 \\
\hline & Bachillerato & 15 & 4.00 & 3.66 & 3.93 & 4.13 & 4.13 & 4.46 & 3.80 & 3.93 \\
\hline & Licenciatura & 9 & 4.50 & 3.66 & 4.44 & 4.33 & 4.22 & 4.44 & 3.66 & 3.77 \\
\hline & Maestría & 2 & 4.28 & 4.00 & 4.00 & 4.00 & 5.00 & 4.50 & 4.50 & 4.00 \\
\hline \multirow{5}{*}{ Estado civil } & Soltero & 28 & 4.06 & 3.71 & 4.17 & 4.10 & 4.21 & 4.60 & 4.07 & 3.60 \\
\hline & Casado & 110 & 4.37 & 4.30 & 4.30 & 4.40 & 4.61 & 4.77 & 4.40 & 3.82 \\
\hline & Divorciado & 5 & 4.11 & 4.40 & 4.20 & 4.60 & 4.40 & 4.40 & 3.40 & 3.40 \\
\hline & Viudo & 5 & 3.54 & 2.80 & 3.80 & 3.80 & 3.80 & 4.40 & 3.60 & 2.60 \\
\hline & Unión libre & 5 & 3.48 & 3.40 & 3.80 & 3.60 & 3.40 & 4.20 & 3.60 & 2.40 \\
\hline \multirow{3}{*}{$\begin{array}{c}\text { \# de visitas en } \\
6 \text { meses }\end{array}$} & $1-3$ & 126 & 4.25 & 4.15 & 4.23 & 4.30 & 4.47 & 4.72 & 4.29 & 3.65 \\
\hline & $4-9$ & 23 & 4.18 & 3.95 & 4.30 & 4.34 & 4.43 & 4.56 & 3.95 & 3.73 \\
\hline & 10 o más & 4 & 4.39 & 4.00 & 4.25 & 4.25 & 4.50 & 4.75 & 4.75 & 4.25 \\
\hline \multirow[t]{3}{*}{ Días estancia } & $1-3$ & 28 & 4.42 & 4.57 & 4.39 & 4.53 & 4.57 & 4.71 & 4.21 & 3.96 \\
\hline & 30 mas & 125 & 4.21 & 4.02 & 4.21 & 4.26 & 4.44 & 4.69 & 4.26 & 3.62 \\
\hline & \multicolumn{3}{|c|}{ Indice de satisfacción del familiar } & 4.12 & 4.24 & 4.31 & 4.47 & 4.69 & 4.25 & 3.68 \\
\hline
\end{tabular}

Resultados de estudios relacionados como el de Jiménez y Villegas M, et (2003) y Ortiz Espinoza R, et (2003) donde se reporta un grado de insatisfacción del $15 \%$ al $28 \%$, con respecto a la evaluación en los servicios de hospitalización, son similares a los encontrados en este estudio realizado en la UMAE $N^{\circ} 1$ Bajío donde se obtuvo un grado de insatisfacción del 23.5\%. Obteniendo $\square \square \square \square \square 3.85$ una $D E=0.636$. Tabla \#4 
Calificación global sobre la Percepción del familiar acompañante con respecto al cuidado de Enfermería

\begin{tabular}{|c|c|c|c|}
\hline Clasificación & $f$ & $\%$ & $\begin{array}{l}\text { Porcentaje } \\
\text { acumulado }\end{array}$ \\
\hline 1.- Inadecuada & 0 & 0 & \multirow{3}{*}{$\begin{array}{c}23.5 \% \\
\text { Insatisfacción }\end{array}$} \\
\hline 2.- No realizado & 8 & 5.2 & \\
\hline 3.- Insuficiente & 28 & 18.30 & \\
\hline 4.- Suficiente & 110 & 71.90 & \multirow{3}{*}{$\begin{array}{c}76.5 \% \\
\text { Satisfacción }\end{array}$} \\
\hline 5.- Excelente & 7 & 4.6 & \\
\hline Total & 153 & 100 & \\
\hline
\end{tabular}

Fuente: IPMSP

$n=153$

\section{DISCUSIÓN}

El cuidado que otorga el personal de Enfermería en los servicios de hospitalización de la UMAE \#1 Bajío en la actualidad es uno de temas de mayor importancia para identificar el trato digno, señalado como índice de calidad de la atención en las unidades de salud, factor importante que nos da pauta para la realización de programas de mejoras en el otorgamiento cada vez de una mejor calidad de atención.

En el presente estudio se indagó sobre el efecto que tiene la percepción del familiar acompañante con respecto a la atención que otorga el personal de enfermería en el Hospital UMAE \#1, con relación a la oportunidad con la presta sus servicios, así como la amabilidad con la que son atendidos, otro punto analizado fue el del proceso de la atención otorgada por enfermería, además de la relación enfermera-paciente, y también se tomó en consideración la información otorgada y la comodidad con que prestó esta atención el personal de enfermería, durante su estancia hospitalaria. Estudios relacionados como el de Jiménez y Villegas (2003), realizado en un hospital del IMSS, reportó un $28 \%$ de insatisfacción en la atención de enfermería, lo cual en nuestro hospital se presentó en $23.5 \%$, otro estudio realizado por Ortiz Espinoza $R$, et al ,2003 reportó cifras más bajas con respecto a la insatisfacción en la atención de enfermería 15\%. Se encontró que la edad promedio de la población oscilaba entre los 31 y 50 años, esto representó el $50.3 \%$ de los participantes $(f=77)$, cabe señalar que no se encontró relación para percibir la calidad de atención de enfermería por el familiar acompañante. Por otro lado el $71.9 \%$ eran casados $(f=110)$. Con relación al nivel educativo un $41.2 \% \quad(f=63)$ señaló contar con un nivel de primaria seguido por un $34.6 \%(f=53)$ para el nivel de secundaria; se encontró que en el estado civil, los familiares acompañantes que se encontraban casados percibieron que la atención de enfermería fue satisfactoria reportando $X^{2} 6.01$ con una $P=.01$.

\section{CONCLUSIONES}

En general conocer cómo percibe el familiar acompañante el cuidado de Enfermería, permite encontrar soluciones y aumentar nuestra calidad de servicio. Los resultados encontrados en este estudio son similares a los estudios relacionados encontrados sobre la atención de enfermería, sin embargo tenemos el reto de lograr un verdadero cambio, que se manifieste 
en una calidad de servicio y que tanto el familiar acompañante como el paciente, manifiesten confianza y seguridad en nuestro cuidado, identificándonos como profesionales de la salud.

El cuidado de enfermería puede percibirse en la respuesta humana del paciente y familiar acompañante y ellos esperan un trato digno por todo el personal multidisciplinario de salud, es por eso que debemos estar alertas a proporcionar cuidados de calidad al paciente y esto se ve reflejado en la satisfacción del familiar.

Las dimensiones de amabilidad, trato personal y oportunidad o tiempos largos de espera por la atención, tiene un alto porcentaje de modificar la percepción del familiar.

El área más afectada en la atención en los servicios de hospitalización de la UMAE N¹ Bajío, es la comodidad, factor importante para el bienestar del paciente, cabe mencionar que esta actividad es una responsabilidad compartida con el equipo multidisciplinario del hospital.

\section{Recomendaciones}

En base a los hallazgos se recomienda realizar otros estudios en los que la muestra de estudio incluya otros hospitales del sector salud, que permita una mejor representación de la población así como de la percepción sobre la atención de enfermería en diferentes hospitales.

También se recomienda que para futuros estudios sobre la atención de enfermería se utilicen diversos instrumentos para lograr capturar mayor información sobre la población y su percepción sobre los diferentes factores que señalen de qué manera se otorga esta atención en los hospitales.

Al igual como indagar sobre las posibles direcciones de asociaciones adicionales entre la percepción del familiar acompañante y las diferentes variables.

\section{REFERENCIAS}

1.- Hernández-Herrera DE y Flores-Hernández S. Relación de ayuda: intervención de Enfermería para padres de recién nacidos hospitalizados en la UCIN. Rev. Enferm IMSS. (2002) 10 (3): 125 - 129.

2.- Gutiérrez-Nava A. La calidad en el servicio. Rev. Enferm IMSS. (2004). 12 (1): 1 - 2.

3.- Marrufo Valles M. Elementos curriculares de la licenciatura en Enfermería. Rev. Enferm IMSS. (2000). 8(1): $45-49$.

4.- Landeros-Olvera E., Salazar-Gómez. Reflexiones sobre el cuidado. Rev. Enferm IMSS. (2006). 14(3): $121-122$.

5.- Ortega Veitía T., de la Cuesta Freijomil D., Días Rutereta C. Propuesta de un instrumento para la aplicación de un proceso de atención de Enfermería en familias disfuncionales. Rev. Cubana Enferm. (1999). 15(3): 164 - 168.

6.- Ramirez-Sanchez TS, Nájera-Aguilar P., Nigenda-Lopez G., Donabedian A. La dimensión internacional de la evaluación y garantía de la calidad. Salud Pública de Mex 1990; 32(2): $113-117$.

7. - Schulz R, O’Brien AT, Bookwala J, Fleisner K. Psychiatric and physical morbidity effects of de dementia caregiving: prevalence correlates and causes. Gerontologist. 1995. 35: 771 791. 
8.- Marriner-Tomey A. Modelo y teorías de enfermería, 3a․ Edición; España: Harcourt Brace 1994; pp.: $341-65$.

9.- López Marure E. Vargas León R. La comunicación interpersonal en I relación enfermerapaciente. Rev. Enferm IMSS. (2002). 10(2): 93 - 102.

10.- Novaes MA, Knobel E, Bork AM, Pavao OF, Nogueira-Martins LA, Ferraz M. S.Tressors In ICU: perception of de patient, relatives and health care team. Intensive Care Med. (1999); 25:1421-6.

11.- Ochoa RV., Lee G M, Arroyo de Cordero G, Jiménez Sánchez J, Terrazas Sotelo L, Camacho Villicaña B, Briseño Piña MD, Flores Soria ME, Carmona Mejía B, Garnica Fonseca R, Gutiérrez Meléndez L, Gutiérrez Méndez J, Reyes Gómez E, Rubio Domínguez S, Suarez Vázquez MG, Sánchez González JM, Hernández Gamboa L, Campos Castolo M, Tena Tamayo C. Recomendaciones para mejorar la atención de enfermería. Rev. Enferm IIMSS. (2004). 12(1): 49-56

12.- Ponce-Gómez J, Reyes-Morales H, Ponce-Gómez G. Satisfacción laboral y calidad de atención de enfermería en una unidad médica de alta especialidad, Rev Enferm IMSS 2006; 14 (2): $65-73$

13.-Jimenez y Villegas Lic. Enf. María del Carmen, Ortega Vargas Lic. Enf. María Carolina, Cruz Ayala Lic. Enf. Gloria, Cruz Corchado Lic. Enf. Maricela, Quintero Barrios Lic. Enf. Martha María, Mendoza Romero Enf. Card. Evelia, Antúnez de la Rosa Enf. Cardiológica María del Roció, Solís Pérez Enf. Car. María Teresa y Hernández Treviño E.A.S.E Martha Elena. Satisfacción del usuario como indicador de calidad. Revista mexicana de enfermería cardiológica. (2003); 11(2) pag.40-41.

14.- Ortiz-Espinoza R, Muñoz-Juárez S, Lechuga- Martín del Campo D, Torres-Carreño E. Consulta externa en instituciones de salud de Hidalgo, México, según la opinión de los usuarios. Rev. Panamericana de Salud Pública 2003; 13(4):229-238. 